\title{
A Periodic Solution of the Generalized Forced Liénard Equation
}

\author{
Zahra Goodarzi and Abdolrahman Razani \\ Department of Mathematics, Faculty of Science, Imam Khomeini International University, Qazvin 34149-16818, Iran \\ Correspondence should be addressed to Zahra Goodarzi; zgoodarzig@gmail.com
}

Received 27 February 2014; Revised 23 May 2014; Accepted 26 May 2014; Published 18 June 2014

Academic Editor: Wing-Sum Cheung

Copyright (C) 2014 Z. Goodarzi and A. Razani. This is an open access article distributed under the Creative Commons Attribution License, which permits unrestricted use, distribution, and reproduction in any medium, provided the original work is properly cited.

We consider the generalized forced Liénard equation as follows: $\left(\phi_{p}\left(x^{\prime}\right)\right)^{\prime}+\left(f(x)+k(x) x^{\prime}\right) x^{\prime}+g(x)=p(t)+s$. By applying Schauder's fixed point theorem, the existence of at least one periodic solution of this equation is proved.

\section{Introduction}

Liénard equation is a kind of differential equations which has a broad set of applications in physics, engineering, and so forth. The existence of at least one periodic solution of this equation has been studied by a number of authors (see, e.g., [1-6]). Forced Liénard equation can be considered as an important generalization of Liénard equations. This one appears in a number of physical models such as fluid mechanics and nonlinear elastic mechanical phenomena.

In this paper, we deal with a generalized forced Liénard equation:

$$
\left(\phi_{p}\left(x^{\prime}\right)\right)^{\prime}+\left(f(x)+k(x) x^{\prime}\right) x^{\prime}+g(x)=p(t)+s,
$$

where $f, k$, and $g$ are real functions on $\mathbb{R}$ such that $g \in$ $\mathrm{C}^{1}\left(\left[R_{1}, R_{2}\right], \mathbb{R}\right), p$ is a $T$-periodic real function on $[0, T]$, $T>0$, and $\phi_{p}: \mathbb{R} \rightarrow \mathbb{R}$ is an increasing homeomorphism with $\phi_{p}(0)=0$ such that $p>1$ and $s \in \mathbb{R}$. Actually, we show that the methodology of [5] can be adopted for more general classes of forced Liénard equations. The application of these equations is in physical models such as classical forced pendulum equations. As an example, consider the following forced pendulum equation:

$$
x^{\prime \prime}+c x^{\prime}+a \sin (x)=h(t)(=\bar{h}+\widetilde{h}(t))
$$

where $c \geq 0, a>0$, and $h(t)=\bar{h}+\widetilde{h}(t)$ is $T$-periodic function for $T>0$, where $\bar{h}$ is the mean value of $h$ and $\widetilde{h}(t)$ has the property $(1 / T) \int_{0}^{T} \widetilde{h}(t) d t=0$. By taking $f(x)=c, k(x)=0$, and $\phi_{p}\left(x^{\prime}\right)=x^{\prime}$, (2) can be considered as an example of (1). This problem is investigated in [7] (see [5] for another example).

\section{Existence of a Periodic Solution}

In this section, we state and prove the existence of at least one periodic solution of the generalized forced Liénard equation by using Schauder's fixed point theorem (see [8-10]). In order to do this, at first consider the following generalized forced Liénard equation:

$$
\left(\phi_{p}\left(x^{\prime}\right)\right)^{\prime}+\left(f(x)+k(x) x^{\prime}\right) x^{\prime}+g(x)=p(t)+s .
$$

Suppose that $f, k$, and $g$ are real functions on $\mathbb{R}$ which are locally Lipschitz such that $g \in \mathbf{C}^{1}\left(\left[R_{1}, R_{2}\right], \mathbb{R}\right)$ and $g^{\prime}(x)>0$. Also, suppose that $p$ is a nonconstant, continuous, and real function on $[0, T]$. Suppose that $M_{0}$ is the maximum value of $|p|$ on $[0, T]$, and $M_{1}, M_{2}$, and $M_{3}$ are the maximum values of $|f|,|g|$, and $|k|$ on $\left[R_{1}, R_{2}\right]$, respectively. Also, suppose that $(1 / T) \int_{0}^{T} p(t) d t=0$. Actually, we describe the set of mean values $s$ for which (3) has at least one $T$-periodic solution. 
Similar to [5], choose a new variable $y=g(x)-s$; then, (3) can be written as follows:

$$
\begin{aligned}
& \left(\phi_{p}\left(\frac{y^{\prime}}{g^{\prime}\left(g^{-1}(y+s)\right)}\right)\right)^{\prime} \\
& \quad+\left(f\left(g^{-1}(y+s)\right)+k\left(g^{-1}(y+s)\right) \frac{y^{\prime}}{g^{\prime}\left(g^{-1}(y+s)\right)}\right) \\
& \quad \times \frac{y^{\prime}}{g^{\prime}\left(g^{-1}(y+s)\right)}+y(t)=p(t) .
\end{aligned}
$$

We now integrate (4) to have

$$
\begin{aligned}
\phi_{p}\left(\frac{y^{\prime}}{g^{\prime}\left(g^{-1}(y+s)\right)}\right) \\
=\int_{0}^{t} p(\tau)-y(\tau)-f\left(g^{-1}(y+s)\right) \frac{y^{\prime}}{g^{\prime}\left(g^{-1}(y+s)\right)} \\
\quad-k\left(g^{-1}(y+s)\right)\left(\frac{y^{\prime}}{g^{\prime}\left(g^{-1}(y+s)\right)}\right)^{2} d(\tau)+C,
\end{aligned}
$$

where $C$ is a constant. $S$ by

In the next step, for all $t \in[0, T]$, we define the operator

$$
\begin{aligned}
S[y](t)=\int_{0}^{t} y(\tau)-p(\tau)+f\left(g^{-1}(y+s)\right) \frac{y^{\prime}}{g^{\prime}\left(g^{-1}(y+s)\right)} \\
+k\left(g^{-1}(y+s)\right)\left(\frac{y^{\prime}}{g^{\prime}\left(g^{-1}(y+s)\right)}\right)^{2} d(\tau) .
\end{aligned}
$$

So by (6), (5) can be written as follows:

$$
y^{\prime}=g^{\prime}\left(g^{-1}(y+s)\right) \phi_{p}^{-1}(-S[y](t)+C) .
$$

One now integrates (7) to get

$$
y(t)=\int_{0}^{t} g^{\prime}\left(g^{-1}(y+s)\right) \phi_{p}^{-1}(-S[y](\tau)+C) d \tau+D,
$$

where $D$ is a constant.

Suppose that $X=\widetilde{C}([0, T], \mathbb{R})$, where $\widetilde{C}$ stands for $T$ periodic continuous functions with zero mean value and

$$
\|y\|=\max _{[0, T]}|y(t)| .
$$

Note that $(X,\|\cdot\|)$ is a Banach space. Now, define the set $\Omega$ as follows:

$$
\Omega=\left\{y \in X: y(t) \in\left[g\left(R_{1}\right)-s, g\left(R_{2}\right)-s\right]\right\} .
$$

One can show that $\Omega$ is a closed, bounded, and convex subspace of $X$. Define the operator $\Phi: \Omega \rightarrow X$ by

$$
\Phi[y](t)=\int_{0}^{t} g^{\prime}\left(g^{-1}(y+s)\right) \phi_{p}^{-1}(-S[y](\tau)+C) d \tau+D .
$$

First, by Lemma 1 of [5], there exists a unique choice of $C$ and $D$, such that $\Phi[y](t) \in X$. Now, we prove that $\Phi: \Omega \rightarrow \Omega$. In order to show this, let $M_{0}$ be the maximum value of $|p|$ on $[0, T]$, and let $M_{1}, M_{2}$, and $M_{3}$ be the maximum values of $|f|$, $|g|$, and $|k|$ on $\left[R_{1}, R_{2}\right]$, respectively. Using (6), we have

$$
\begin{gathered}
\mid \int_{0}^{t} y(\tau)-p(\tau)+f\left(g^{-1}(y+s)\right) \frac{y^{\prime}}{g^{\prime}\left(g^{-1}(y+s)\right)} \\
+k\left(g^{-1}(y+s)\right)\left(\frac{y^{\prime}}{g^{\prime}\left(g^{-1}(y+s)\right)}\right)^{2} d(\tau) \mid \\
\quad \leq T\left(\left|g\left(R_{2}\right)-s\right|+M_{0}+M_{1} r+M_{3} r^{2}\right),
\end{gathered}
$$

where $r=\max _{[0, T]}\left|x^{\prime}(t)\right|$. Hence, for all $t \in[0, T]$

$$
|S[y](t)| \leq T\left(\left|g\left(R_{2}\right)-s\right|+M_{0}+M_{1} r+M_{3} r^{2}\right),
$$

so

$$
\begin{aligned}
- & \left(T\left(\left|g\left(R_{2}\right)-s\right|+M_{0}+M_{1} r+M_{3} r^{2}\right)+C\right) \\
& \leq S[y](t)-C \\
& \leq T\left(\left|g\left(R_{2}\right)-s\right|+M_{0}+M_{1} r+M_{3} r^{2}\right)-C .
\end{aligned}
$$

Also, we have

$$
\begin{gathered}
-\left|T\left(\left|g\left(R_{2}\right)-s\right|+M_{0}+M_{1} r+M_{3} r^{2}\right)+C\right| \leq S[y](t)-C \\
\leq\left|T\left(\left|g\left(R_{2}\right)-s\right|+M_{0}+M_{1} r+M_{3} r^{2}\right)-C\right| .
\end{gathered}
$$

Set

$$
\begin{gathered}
A:=-\left|T\left(\left|g\left(R_{2}\right)-s\right|+M_{0}+M_{1} r+M_{3} r^{2}\right)+C\right|, \\
B:=\left|T\left(\left|g\left(R_{2}\right)-s\right|+M_{0}+M_{1} r+M_{3} r^{2}\right)-C\right| .
\end{gathered}
$$

Hence, for each $y \in \Omega$ and $t \in[0, T]$, we have

$$
A \leq S[y](t)-C \leq B
$$

Using the same method of [5], one can prove the existence of at least one $T$-periodic solution of (1) as follows.

Theorem 1. Suppose that $f, k$, and $g$ are real functions on $\mathbb{R}$ which are locally Lipschitz such that $g \in \mathbf{C}^{1}\left(\left[R_{1}, R_{2}\right], \mathbb{R}\right)$ and $g^{\prime}(x)>0$ for real numbers $R_{1}$ and $R_{2}$ in which $R_{1}<R_{2}$, $p$ is a nonconstant, continuous, and T-periodic real function on $[0, T]$ for $T>0$, and $\phi_{p}: \mathbb{R} \rightarrow \mathbb{R}$ is an increasing homeomorphism with $\phi_{p}(0)=0$, and $s \in \mathbb{R}$. Let $\Phi, \Omega, A$, and $B$ be defined by (11), (10), and (16), respectively. If

$$
\begin{gathered}
s \in\left[g\left(R_{1}\right)+T \max _{\left[R_{1}, R_{2}\right]}\left|g^{\prime}(x)\right| \phi_{p}^{-1}(B-A)+|D|,\right. \\
\left.g\left(R_{2}\right)-T \max _{\left[R_{1}, R_{2}\right]}\left|g^{\prime}(x)\right| \phi_{p}^{-1}(B-A)-|D|\right], \\
2\left(T \max _{x \in\left[R_{1}, R_{2}\right]}\left|g^{\prime}(x)\right| \phi_{p}^{-1}(B-A)+|D|\right) \leq\left|g\left(R_{1}\right)-g\left(R_{2}\right)\right|,
\end{gathered}
$$

then (3) has at least one T-periodic solution on $\Omega$. 
Proof. According to the definition of $\Phi$ and (17), $\Phi: \Omega \rightarrow X$ is continuous. Hence, in order to use Schauder's fixed point theorem, we need to prove that $\Phi$ is compact and maps $\Omega$ into itself. For $y \in \Omega$ and $t \in[0, T]$, we have

$$
|\Phi[y](t)| \leq T \max _{\left[R_{1}, R_{2}\right]}\left|g^{\prime}(x)\right| \phi_{p}^{-1}(B-A)+|D|,
$$

because

$$
-B \leq-S[y](t)+C \leq-A,
$$

so

$$
0 \leq-S[y](t)+C+B \leq B-A
$$

hence,

$$
-S[y](t)+C \leq-S[y](t)+C+B \leq B-A .
$$

Now, by (18) for all $t \in[0, T]$,

$$
g\left(R_{1}\right)-s \leq \Phi[y](t) \leq g\left(R_{2}\right)-s .
$$

So $\Phi: \Omega \rightarrow \Omega$.

We recall a given sequence $\left\{\phi_{n}(t)\right\}$ of functions from $[a, b]$ to $\mathbb{R}$, which is called equicontinuous if for every $\epsilon>0$ there exists $\delta>0$ such that for all $n \in \mathbb{N}$ and for all $t_{1}, t_{2} \in[a, b]$ if $\left|t_{1}-t_{2}\right|<\delta$ then $\left|\phi_{n}\left(t_{1}\right)-\phi_{n}\left(t_{2}\right)\right|<\epsilon$. Now, we recall the Ascoli-Arzela theorem as follows.

Theorem 2 (Ascoli-Arzela). Let $\phi_{n}(t)$ be a sequence of functions from $[a, b]$ to $\mathbb{R}$ which is uniformly bounded and equicontinuous. Then, $\phi_{n}(t)$ has a uniformly convergent subsequence.

In the sequel, we show that $\Phi$ is a compact operator on $\Omega$. For this, we show that each bounded sequence $\left\{y_{n}\right\}$ in $\Omega$ has a convergent subsequence $\left\{y_{n_{i}}\right\}$ in $\Omega$. Suppose that $\left\{y_{n}\right\}$ is a sequence in $\Omega$. It is clear that $\left\{y_{n}\right\}$ is bounded. Set $\max _{\left[R_{1}, R_{2}\right]}\left|g^{\prime}(x)\right| \phi_{p}^{-1}(B-A)=N$, for given $\epsilon>0$; then, there exists $\delta>0$ such that $0<N \delta<\epsilon$ and, for every $t_{1}, t_{2} \in[0, T]$, if $\left|t_{1}-t_{2}\right|<\delta$, then

$$
\left|\Phi\left[y_{n}\right]\left(t_{1}\right)-\Phi\left[y_{n}\right]\left(t_{2}\right)\right| \leq N\left|t_{1}-t_{2}\right|<\epsilon .
$$

So,

$$
\left\|\Phi\left[y_{n}\right]\left(t_{1}\right)-\Phi\left[y_{n}\right]\left(t_{2}\right)\right\|<\epsilon .
$$

Therefore, $\left\{\Phi\left[y_{n}\right](t)\right\}$ is an equicontinuous sequence on $[0, T]$. By the Ascoli-Arzela theorem, there exists a subsequence $\left\{y_{n_{i}}(t)\right\}$ of $\left\{y_{n}(t)\right\}$ such that $\left\{\Phi\left[y_{n_{i}}\right](t)\right\}$ is uniformly convergent on $[0, T]$. Hence, $\Phi$ is a compact operator.

Now, we recall Schauder's fixed point theorem as follows.

Theorem 3 (Schauder's fixed point theorem). Let $X$ be a Banach space and $\Omega$ a closed, bounded, and convex subset of $X$. If $S: \Omega \rightarrow \Omega$ is a compact operator, then $S$ has at least one fixed point on $\Omega$.

Therefore, by Schauder's fixed point theorem, there exists $y \in \Omega$ such that $\Phi(y)=y$. This means that $x=g^{-1}(y+s)$ is a solution of (1).
When $g^{\prime}(x)<0$, Theorem 1 is satisfied by the following changes:

$$
\Omega=\left\{y \in X: y(t) \in\left[g\left(R_{2}\right)-s, g\left(R_{1}\right)-s\right]\right\} .
$$

Suppose that $A, B$ are defined by

$$
\begin{gathered}
A:=-\left|T\left(\left|g\left(R_{1}\right)-s\right|+M_{0}+M_{1} r+M_{3} r^{2}\right)+C\right|, \\
B:=\left|T\left(\left|g\left(R_{1}\right)-s\right|+M_{0}+M_{1} r+M_{3} r^{2}\right)-C\right|,
\end{gathered}
$$

where $r$ is defined by $r=\max _{[0, T]}\left|x^{\prime}(t)\right|$. Now, we have the next corollary.

Corollary 4. Suppose that $f, k$, and $g$ are real functions on $\mathbb{R}$ which are locally Lipschitz such that $g \in \mathbf{C}^{1}\left(\left[R_{1}, R_{2}\right], \mathbb{R}\right)$ and $g^{\prime}(x)<0$ for real numbers $R_{1}$ and $R_{2}$ in which $R_{1}<R_{2}$, $p$ is a nonconstant, continuous, and T-periodic real function on $[0, T]$ for $T>0$, and $\phi_{p}: \mathbb{R} \rightarrow \mathbb{R}$ is an increasing homeomorphism with $\phi_{p}(0)=0$, and $s \in \mathbb{R}$. Let $\Phi, \Omega, A$, and $B$ be defined by (10), (27), and (28), respectively. If

$$
\begin{gathered}
2\left(T \max _{x \in\left[R_{1}, R_{2}\right]}\left|g^{\prime}(x)\right| \phi_{p}^{-1}(B-A)+|D|\right) \leq\left|g\left(R_{1}\right)-g\left(R_{2}\right)\right|, \\
s \in\left[g\left(R_{2}\right)+T \max _{\left[R_{1}, R_{2}\right]}\left|g^{\prime}(x)\right| \phi_{p}^{-1}(B-A)+|D|,\right. \\
\left.g\left(R_{1}\right)-T \max _{\left[R_{1}, R_{2}\right]}\left|g^{\prime}(x)\right| \phi_{p}^{-1}(B-A)-|D|\right],
\end{gathered}
$$

then (3) has at least one T-periodic solution on $\Omega$.

Proof. Set

$$
\Omega=\left\{y \in X: y(t) \in\left[g\left(R_{2}\right)-s, g\left(R_{1}\right)-s\right]\right\} .
$$

Now, one can prove the existence of at least one $T$-periodic solution of (3) by the same argument in the proof of Theorem 1.

Now, we consider a special case $\phi(x)=x$ and prove the existence of at least one $T$-periodic solution of the equation

$$
x^{\prime \prime}+\left(f(x)+k(x) x^{\prime}\right) x^{\prime}+g(x)=p(t)+s
$$

where $f, k$, and $g$ are real functions on $\mathbb{R}$ such that $g \in$ $\mathrm{C}^{1}\left(\left[R_{1}, R_{2}\right], \mathbb{R}\right), p$ is a $T$-periodic real function on $[0, T], T>$ 0 , and $s \in \mathbb{R}$. Consider that $f, k$, and $g$ are real functions on $\mathbb{R}$ such that $g \in C^{1}\left[R_{1}, R_{2}\right]$ and $g^{\prime}(x)>0$. Suppose that $M_{0}$ is the maximum value of $|p|$ on $[0, T]$ and $M_{1}, M_{2}$, and $M_{3}$ are the maximum values of $|f|,|g|$, and $|k|$ on $\left[R_{1}, R_{2}\right]$. Also, suppose that $\phi$ is defined by (11). In the following, it is shown that (31) has at least one $T$-periodic solution. 
Define a new variable $y=g(x)-s$; thus, (31) can be rewritten as follows:

$$
\begin{aligned}
& \left(\frac{y^{\prime}}{g^{\prime}\left(g^{-1}(y+s)\right)}\right)^{\prime} \\
& \quad+\left(f\left(g^{-1}(y+s)\right)+k\left(g^{-1}(y+s)\right) \frac{y^{\prime}}{g^{\prime}\left(g^{-1}(y+s)\right)}\right) \\
& \quad \times \frac{y^{\prime}}{g^{\prime}\left(g^{-1}(y+s)\right)}+y(t)=p(t) .
\end{aligned}
$$

Then,

$$
\begin{aligned}
& \frac{y^{\prime}}{g^{\prime}\left(g^{-1}(y+s)\right)} \\
& \quad=\int_{0}^{t} p(\tau)-y(\tau)-f\left(g^{-1}(y+s)\right) \frac{y^{\prime}}{g^{\prime}\left(g^{-1}(y+s)\right)} \\
& \quad-k\left(g^{-1}(y+s)\right)\left(\frac{y^{\prime}}{g^{\prime}\left(g^{-1}(y+s)\right)}\right)^{2} d(\tau)+C,
\end{aligned}
$$

where $C$ is a constant.

Now, define the operator $S$, for all $t \in[0, T]$, by

$$
\begin{aligned}
& S[y](t) \\
& =\int_{0}^{t} y(\tau)-p(\tau)+f\left(g^{-1}(y+s)\right) \frac{y^{\prime}}{g^{\prime}\left(g^{-1}(y+s)\right)} \\
& \quad+k\left(g^{-1}(y+s)\right)\left(\frac{y^{\prime}}{g^{\prime}\left(g^{-1}(y+s)\right)}\right)^{2} d(\tau) .
\end{aligned}
$$

Also,

$$
y^{\prime}=g^{\prime}\left(g^{-1}(y+s)\right)(-S[y](t)+C) .
$$

So,

$$
y(t)=\int_{0}^{t} g^{\prime}\left(g^{-1}(y+s)\right)(-S[y](\tau)+C) d \tau+D,
$$

where $D$ is a constant.

Consider that $X=\widetilde{C}([0, T], \mathbb{R})$ and $\|\cdot\|$ is defined by (9). Define $\Omega$ by

$$
\Omega=\left\{y \in X: y(t) \in\left[g\left(R_{1}\right)-s, g\left(R_{2}\right)-s\right]\right\} .
$$

$\Omega$ is a closed, bounded, and convex subspace of $X$. Define the operator $\Phi: \Omega \rightarrow X$ by

$$
\Phi[y](t)=\int_{0}^{t} g^{\prime}\left(g^{-1}(y+s)\right)(-S[y](\tau)+C) d \tau+D .
$$

Again, by Lemma 1 of [5], there exists a unique choice of $C$ and $D$, such that $\phi[y](t) \in X$ and with the same argument as before, $\Phi: \Omega \rightarrow \Omega$.

Now, the following corollary proves the existence of at least one $T$-periodic solution for (31) on $\Omega$.
Corollary 5. Suppose that $f, k$, and $g$ are real functions on $\mathbb{R}$ which are locally Lipschitz such that $g \in \mathbf{C}^{1}\left(\left[R_{1}, R_{2}\right], \mathbb{R}\right)$ and $g^{\prime}(x)>0\left(g^{\prime}(x)<0\right)$ for real numbers $R_{1}$ and $R_{2}$ in which $R_{1}<R_{2}, p$ is a nonconstant, continuous, and T-periodic real function on $[0, T]$ for $T>0$, and $s \in \mathbb{R}$. Let $\Omega, A$, and $B$ be defined by (16) and (37), respectively. If

$$
\begin{gathered}
s \in\left[g\left(R_{1}\right)+T \max _{\left[R_{1}, R_{2}\right]}\left|g^{\prime}(x)\right|(B-A)+|D|,\right. \\
\left.g\left(R_{2}\right)-T \max _{\left[R_{1}, R_{2}\right]}\left|g^{\prime}(x)\right|(B-A)-|D|\right], \\
2\left(T \max _{x \in\left[R_{1}, R_{2}\right]}\left|g^{\prime}(x)\right|(B-A)+D\right) \leq\left|g\left(R_{1}\right)-g\left(R_{2}\right)\right|,
\end{gathered}
$$

then (31) has at least one T-periodic solution on $\Omega$.

Proof. Suppose that $\Phi: \Omega \rightarrow X$ is defined by (38). Now, one can prove the existence of at least one $T$-periodic solution of (31) by the same method of Theorem 1 , (case $g^{\prime}(x)<0$ is the same).

Remark 6. A good question is to study the stability of $T$ periodic solution of (1).

\section{Conflict of Interests}

The authors declare that there is no conflict of interests regarding the publication of this paper.

\section{Acknowledgment}

The authors would like to thank the referee for the valuable comments and suggestions.

\section{References}

[1] H. B. Chen, K. T. Li, and D. S. Li, "Existence of exactly one and two periodic solutions of the Liénard equation," Acta Mathematica Sinica, vol. 47, no. 3, pp. 417-424, 2004.

[2] R. Manasevic, J. Mawhin, and F. Zanolin, Periodic Solutions of Complex-Valued Differential Equations and Systems with Periodic Coefficient, SISSA, 1995.

[3] M. R. Pournaki and A. Razani, "On the existence of periodic solutions for a class of generalized forced Liénard equations," Applied Mathematics Letters, vol. 20, no. 3, pp. 248-254, 2007.

[4] R. Srzednicki, "Periodic and bounded solutions in blocks for time-periodic nonautonomous ordinary differential equations," Nonlinear Analysis: Theory, Methods \& Applications, vol. 22, no. 6, pp. 707-737, 1994.

[5] P. J. Torres, "Nondegeneracy of the periodically forced Liénard differential equation with $\varphi$-Laplacian," Communications in Contemporary Mathematics, vol. 13, no. 2, pp. 283-292, 2011.

[6] J. Zhou, S. Sun, and Z. Liu, "Periodic solutions of forced Liénard-type equations," Applied Mathematics and Computation, vol. 161, no. 2, pp. 656-666, 2005. 
[7] J. Mawhin, "Seventy-five years of global analysis around the forced pendulum equation," in Equadiff Proceedings, vol. 9, pp. 115-145, Masaryk University, Brno \& Electronic Publishing House, Stony Brook, 1998.

[8] Z. Goodarzi, M. R. Mokhtarzadeh, and A. Razani, "Existence of at least one periodic solution of the coupled Riccati differential equations," submitted.

[9] M. R. Mokhtarzadeh, M. R. Pournaki, and A. Razani, "An existence-uniqueness theorem for a class of boundary value problems," Fixed Point Theory, vol. 13, no. 2, pp. 583-591, 2012.

[10] M. R. Mokhtarzadeh, M. R. Pournaki, and A. Razani, "A note on periodic solutions of Riccati equations," Nonlinear Dynamics, vol. 62, no. 1-2, pp. 119-125, 2010. 


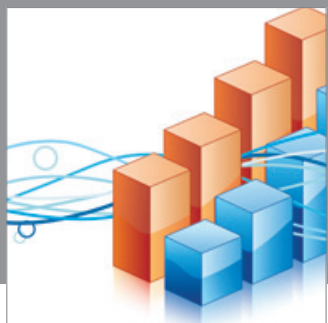

Advances in

Operations Research

mansans

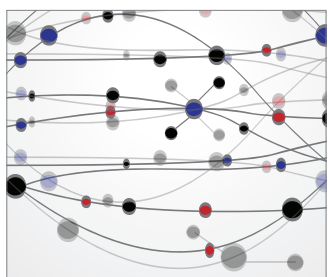

The Scientific World Journal
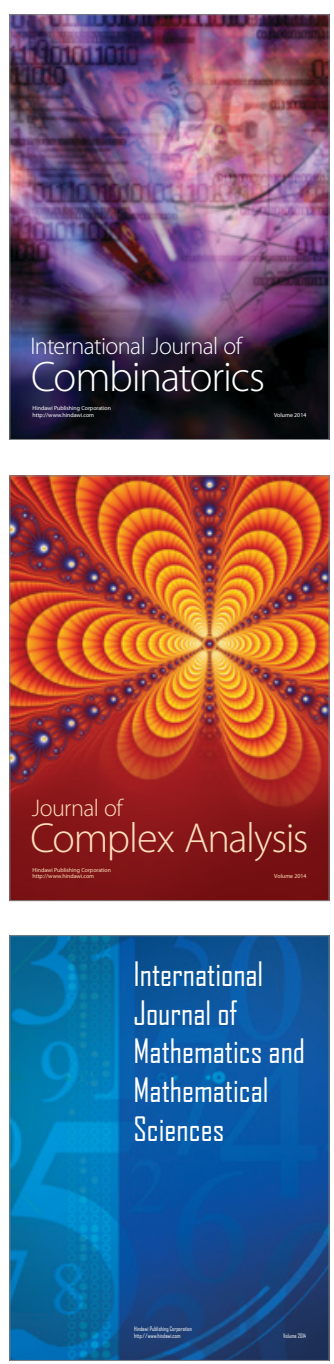
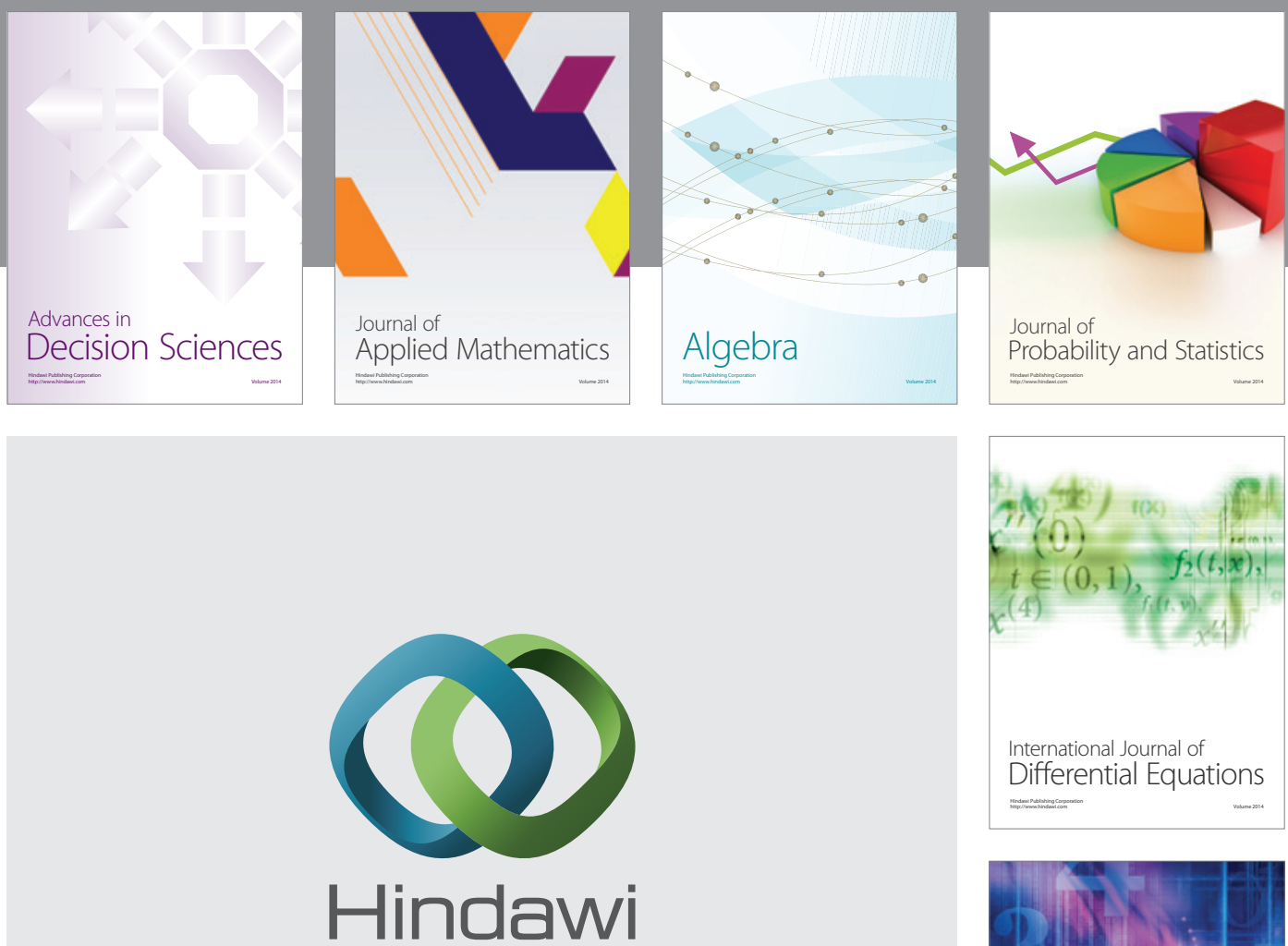

Submit your manuscripts at http://www.hindawi.com
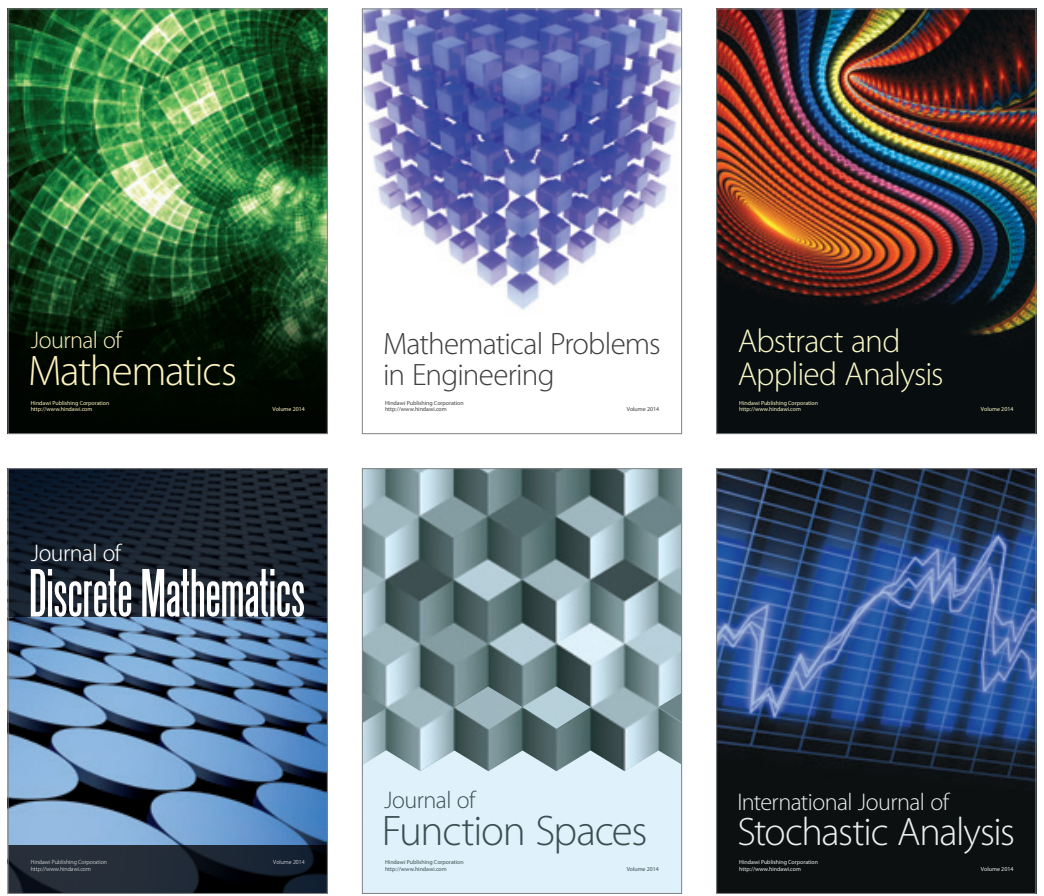

Journal of

Function Spaces

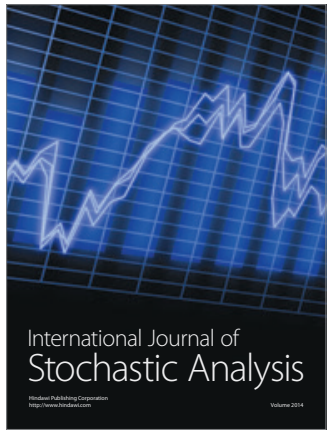

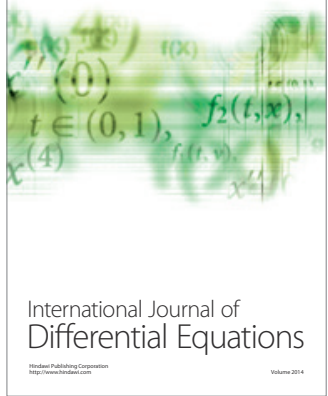
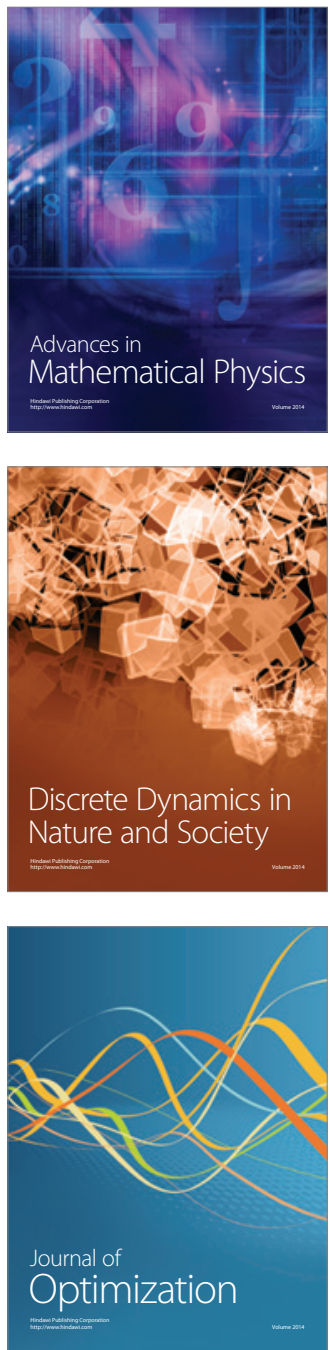